\title{
MODELO DE REFERÊNCIA PARA O DESENVOLVIMENTO DE PRODUTOS DE TECNOLOGIA ASSISTIVA PARA O SETOR DE TRANSPORTE AÉREO
}

\author{
Alexandre Machado Rocha (arocha@usp.br) - Escola Politécnica, Universidade de São Paulo \\ Eduardo de Senzi Zancul (ezancul@usp.br) - Escola Politécnica, Universidade de São Paulo
}

\section{RESUMO}

A demanda por soluções de acessibilidade e de tecnologia assistiva (TA) é crescente frente ao envelhecimento e ao crescimento da população mundial previstos para as próximas décadas. Especificamente no setor de transporte aéreo, acessibilidade e soluções de TA são questões críticas e, muitas vezes, complexas de serem adotadas devido a fatores como, por exemplo, os espaços restritos das aeronaves, normas rígidas dos órgãos de aviação, questões de segurança de voo, e envolvimento de diversos stakeholders. Ao mesmo tempo, no campo de desenvolvimento de produtos, há poucas pesquisas em relação ao desenvolvimento de soluções de tecnologia assistiva. $\mathrm{O}$ objetivo deste trabalho é propor um modelo de referência para o desenvolvimento conceitual de produtos de tecnologia assistiva para o setor de transporte aéreo. O trabalho analisa projetos de pesquisa de desenvolvimento de soluções de TA para o setor de transporte aéreo como base para a criação do modelo, que utiliza o design thinking como abordagem em projetos. $\mathrm{O}$ modelo de referência proposto pode ser futuramente testado e adaptado para o desenvolvimento de TA em outros setores, ou ainda ser expandido e utilizado como base para aplicação fora do ambiente de pesquisa no contexto de indústria.

Palavras chave: Processo de desenvolvimento de produtos; Tecnologia assistiva; Design thinking; Setor de transporte aéreo; Modelo de referência.

Área: Gestão do Processo de Desenvolvimento de Produtos 


\section{INTRODUÇÃO}

A necessidade por soluções de tecnologia assistiva vem aumentando nas últimas décadas como consequência do aumento da expectativa de vida da população e também do seu crescimento e envelhecimento, tanto a nível mundial quanto a nível nacional. Segundo Hersh (2010), pessoas com deficiência e/ou pessoas idosas são os usuários principais de tecnologias assistivas, e seus requisitos devem ser levados em consideração no design de produtos de TA.

Diversos setores da economia são impactados com aumento da demanda da população e aumento das exigências. No setor de transporte aéreo, por exemplo, o envelhecimento da população por si só representa uma tendência de aumento no número de passageiros com maior idade (RAYMAN, 1997), ao mesmo tempo em que fatores como aeronaves mais modernas e mais eficientes no consumo de combustíveis e menores custos de operação também incentivam a expansão do mercado. Entre os anos de 2004 e 2015, o número de passageiros transportados por ano subiu de 2 bilhões para 3,5 bilhões de passageiros em 100.000 voos diários, ou seja, um aumento de 75\% em apenas uma década (IATA, 2016).

Considerando o ambiente de uma aeronave, a acessibilidade se torna crítica especialmente devido a restrições de peso, de espaço, e a procedimentos de segurança, resultando em dificuldades no acesso de pessoas com diversas deficiências ou limitações, não apenas físicas, mas também cognitivas. Fatores como esses tornam complexo o desenvolvimento de soluções de acessibilidade também no setor de transporte aéreo.

Assim sendo, a necessidade de soluções para idosos e pessoas com deficiência leva a oportunidades para apoio ao desenvolvimento de produtos de tecnologia assistiva. Especificamente no contexto brasileiro, o mercado de tecnologia assistiva é ocupado por poucas empresas, maioria delas estrangeiras, que importam soluções para o mercado nacional. Consequentemente os produtos possuem custos elevados e impeditivos que restringem o acesso de uma grande parcela da população.

Por parte do Governo brasileiro, nas últimas décadas tem havido incentivos associados ao Plano Nacional dos Direitos da Pessoa com Deficiência, em conjunto com financiamentos de projetos de pesquisa e de inovação por meio de órgãos governamentais como CNPq, CAPES e FINEP.

Nesse contexto, a pergunta de pesquisa que incentiva este trabalho é como facilitar e/ou aperfeiçoar o desenvolvimento de projetos de produtos de tecnologia assistiva em centros de pesquisa e instituições de ensino superior?

Este trabalho foca no contexto da acessibilidade no setor de transporte aéreo, analisando casos de projetos de pesquisa para o desenvolvimento de produtos de TA para aeronaves. $\mathrm{O}$ trabalho apresenta a proposta de um modelo de referência para o desenvolvimento conceitual de produtos de tecnologia assistiva para o setor de transporte aéreo utilizando abordagem de design thinking.

\section{REVISÃO BIBLIOGRÁFICA}

O conteúdo da revisão de literatura deste artigo engloba tópicos relacionados a tecnologia assistiva, desenvolvimento de produto e setor de transporte aéreo. Primeiramente é apresentado o conceito de tecnologia assistiva e design universal, seguido por uma avaliação sobre processos de desenvolvimento de produto, passando por design thinking e por informações do setor de transporte aéreo. 
Para a revisão bibliográfica de tecnologia assistiva e de desenvolvimento de produto foi utilizada a base de dados ISI Web of Knowledge, e também buscas na ferramenta Google Acadêmico.

A pesquisa realizada inicialmente na base de dados ISI Web of Knowledg e utilizou os termos "product development" e "assistive" ou "inclusive" ou "rehabilitation" - strings utilizadas: "product development" AND ("assistive" OR "inclusive" OR "rehabilitation"). O resultado da busca revelou uma lista com 82 artigos em Junho/2015 (atualizada para 89 artigos em dezembro/2016). Após análise dos títulos e dos resumos dos artigos, foi possível concluir que nenhum deles tratava do processo pleno de desenvolvimento de produto.

Em seguida, foi realizada uma busca no site de pesquisas Google Acadêmico com a finalidade de expandir a base de dados. A busca foi realizada com os termos "product development" $\mathrm{e}$ "assistive", e dentre os resultados obtidos foram encontrados artigos, livros sobre tecnologia assistiva e publicações de conferências. Além dos resultados das buscas no ISI e no Google Acadêmico, outras referências foram encontradas a partir de leitura do material encontrado.

\subsection{Tecnologia Assistiva e Design Universal}

O termo tecnologia assistiva é bastante utilizado na literatura, mas nenhuma definição é conclusiva e totalmente aceita (COOK; POLGAR, 2008; HERSH, 2010). É possível encontrar diversas definições para o termo, todas muito similares.

Para fins deste trabalho, é considerada a definição adotada pelo Governo Brasileiro: "Tecnologia Assistiva é uma área do conhecimento, de característica interdisciplinar, que engloba produtos, recursos, metodologias, estratégias, práticas e serviços que objetivam promover a funcionalidade, relacionada à atividade e participação, de pessoas com deficiência, incapacidades ou mobilidade reduzida, visando sua autonomia, independência, qualidade de vida e inclusão social" (BRASIL - SDH/PR, 2009).

Vinculado à tecnologia assistiva está o termo design universal, ou design for all (design para todos) como é disseminado na Europa, que é uma abordagem cujo objetivo é criar produtos acessíveis e utilizáveis pelo maior número de pessoas possível, independente de deficiência, idade, sexo, ou classe social (HERSH, 2010).

Design universal pode ser definido como design de ambientes ou produtos que pode ser utilizado por pessoas de qualquer idade ou habilidade sem a necessidade de adaptações. E quando bem aplicado, se adapta tão bem ao ambiente ou produto que se torna "invisível" (MACE, 1998).

\subsection{Processo de desenvolvimento de produtos}

Considerando os esforços para o desenvolvimento de tecnologias assistivas, o tema de desenvolvimento de produto passa a ser relevante.

O objetivo de qualquer projeto de desenvolvimento de produto ou de serviço é transformar uma ideia em realidade, desde o conceito até o produto específico que atenda as necessidades de mercado. Assim, o processo de desenvolvimento de produto ou de serviço permite uma organização transformar oportunidades de mercado e questões técnicas em produtos ou serviços com resultados comerciais (CLARK; FUJIMOTO, 1992).

Com o objetivo de apresentar um modelo que contenha as melhores práticas em desenvolvimento de produtos, Rozenfeld et al. (2006) propõem o chamado Modelo Unificado do PDP, que tomou como base o desenvolvimento de bens duráveis e de equipamentos, mas, segundo os próprios autores, pode ser adaptado para o desenvolvimento de outros tipos de 
produtos. O modelo é composto de três macro fases e nove fases, cada uma delas com fluxos detalhados de atividades e tarefas. As três macro fases são Pré-desenvolvimento, Desenvolvimento e Pós-desenvolvimento, que incluem as fases de Planejamento Estratégico dos Produtos, Planejamento do Projeto, Projeto Informacional, Projeto Conceitual, Projeto Detalhado, Preparação para Produção, Lançamento do Produto, Acompanhar Produto/Processo, e Descontinuar Produto.

Há diversas obras disponíveis na literatura que tratam de adequar um modelo de PDP a cenários ou indústrias específicas por meio da criação de modelos de referência específicos. De acordo com uma pesquisa realizada no Brasil entre os anos de 2001 e 2011 por meio da lista de programas de pós-graduação da ABEPRO (Associação Brasileira de Engenharia de Produção) foram realizados 32 trabalhos sobre modelos para o PDP (MORETTI, 2012). Contudo, não foi encontrado nenhum modelo referente a produtos de tecnologia assistiva.

Por fim, foram identificados quatros trabalhos que tocam na questão do processo de desenvolvimento de produto de tecnologias assistivas: Cook \& Polgar (2008), Hersh (2010), Okumura e Canciglieri Júnior (2011) e Flagg et al. (2013).

O denominado de modelo de tecnologia assistiva para atividade humana (HAAT, Human Activity Assistive Technology model, em inglês) tem como objetivo o entendimento do posicionamento da tecnologia assistiva na vida de pessoas com deficiência, guiando aplicações clínicas e pesquisas investigativas, com foco no componente humano, na tecnologia e nos contextos (social, cultural, físico e institucional). As atividades (lazer, pessoais, produtivas) em conjunto com os contextos de uso e as habilidades da PcD determinam as características da tecnologia assistiva (COOK; POLGAR, 2008). Contudo, o HAAT não tem como foco o processo de desenvolvimento de produto, mas sim a forma como ele é aplicado. Por isso, esse e outros trabalhos são chamados de "conceptual models for assistive technology outcomes", algo como modelos conceituais para resultados de tecnologia assistiva, com foco em aplicação dos produtos, especialmente na relação terapeuta-paciente.

Hersh (2010) se aprofunda no design e avaliação de produtos de tecnologia assistiva, e mostra a relação dos produtos de tecnologia assistiva com produtos de reabilitação, produtos médicos, e produtos em geral, fazendo a distinção entre eles. Além disso, apresenta algumas semelhanças e diferenças entre tais produtos e aqueles de tecnologia assistiva. Características como facilidade e intuitividade de uso para atender às necessidades específicas do usuário, e estar de acordo com normas e legislações nacionais e internacionais são consideradas.

Okumura e Canciglieri Junior (2011) propõe uma abordagem para o desenvolvimento de produto inclusivo no ambiente de engenharia simultânea. Os autores propõem um processo de desenvolvimento integrado de produtos com base no conceito de engenharia simultânea, em conjunto com métodos e ferramentas do PDP e considerando questões de acessibilidade, TA, e desenho universal, com o intuito de atender um número maior de usuários. Recomendam o envolvimento de profissionais de várias áreas no processo de desenvolvimento do produto como forma de desenvolver um produto da TA mais eficaz no atendimento da necessidade da PcD. Afirmam que o uso dos conceitos da engenharia simultânea no processo para desenvolvimento dos dispositivos da TA resulta em menor prazo de fabricação e contribui para um produto com melhor qualidade e menor custo.

Flagg et al. (2013) mostram o modelo chamado Need to Knowledge (NtK) Model, uma abordagem prática e orientada ao mercado com o objetivo de permitir o retorno do investimento público em pesquisa e inovação na forma de benefícios econômico-sociais. $\mathrm{O}$ Modelo NtK surgiu nos EUA a partir da percepção de que os investimentos em pesquisa na área de tecnologia assistiva tinham dificuldade de chegar ao mercado e gerar como resultado 
produtos comerciais. O modelo considera o processo de inovação em três níveis: nível de pesquisa que gera conceitos, nível de desenvolvimento e engenharia que gera protótipos, e nível industrial que gera inovação comercial.

\subsection{Abordagem centrada no usuário e Design Thinking}

Kutz (2003) comenta que os engenheiros projetistas tradicionais tendem a focar na função do produto, dando pouca importância à forma e usabilidade, enquanto o oposto poderia levar a um design de produto mais acessível para pessoas com deficiência, produzindo como resultado um produto melhor para todos e com potencial de aumento de market share. Nesse sentido se encaixa a abordagem do design thinking, que apresenta atividades de inovação centradas no ser humano (human-centered design).

O design thinking é inspirado na forma de trabalhar da área do design como uma abordagem metodológica para ser utilizada por profissionais de outras áreas além do design. Tim Brown (2008) denomina o design thinking como sendo uma metodologia que permeia todo o espectro das atividades de inovação, com um design centrado no ser humano (human-centered design). $\mathrm{O}$ autor sintetiza o design thinking como uma disciplina que utiliza da sensibilidade do designer e de métodos para atender às necessidades das pessoas com o que é tecnologicamente e economicamente viável se convertendo em valor para o cliente e oportunidade de mercado.

A abordagem proposta por Brown (2008) é centrada no usuário, e moldada em um formato cíclico dividido em três grandes etapas: inspiração, quando são feitas pesquisas para busca de soluções; ideação, para criar e testar ideias e soluções; implementação, que leva em consideração a percepção de mercado e realiza a implementação comercial e industrial, e pode viabilizar o início de um novo ciclo de desenvolvimento.

A abordagem proposta pela d.school de Stanford é chamada Bootcamp Bootleg e é dividida em cinco fases: empatia, definição, ideação, prototipação, e testes (D.SCHOOL, 2009).

Há outras abordagens de design thinking, mas todas as propostas possuem elementos importantes em comum: entendimento do usuário, etapas de ideação e diversos ciclos de prototipagem e testes.

\subsection{Setor de transporte aéreo}

Apenas no ano de 2014 foram transportados mais de 3,2 bilhões de passageiros em todo o mundo, segundo o World Bank Group (2015), com base em dados fornecidos pelas próprias companhias aéreas. No Brasil, a demanda de transporte de passageiros também tem crescido. Entre 2002 e 2014, o volume de passageiros transportados triplicou, alcançando 114 milhões de passageiros ao ano, com a tendência de atingir 211 milhões em 2020 (ABEAR, 2014).

A Agência Nacional de Aviação Civil - ANAC determina as regras que as companhias aéreas devem seguir para garantir a acessibilidade e o acesso de Passageiros com Necessidades de Assistência Especial (PNAE). A Resolução N. ${ }^{\circ} 280$ de 2013 (ANAC, 2013) determina, entre outros pontos, o número mínimo de braços móveis nas poltronas da aeronave e a obrigatoriedade ou não de uma cadeira de rodas para uso no interior da aeronave.

No setor de transporte aéreo, a disponibilidade de ambientes com acessibilidade adequada e a disponibilidade de equipamentos de acessibilidade é limitada, e a aplicação de tecnologias assistivas é complexa devido aos inúmeros stakeholders envolvidos, às restrições de segurança nos aeroportos, a questões logísticas, e às restrições de segurança, espaço e peso nas aeronaves (BARGAR et al., 2014; NAIARA et al., 2015). 
Assim, os usuários de transporte aéreo que necessitam de acessibilidade e utilizam tecnologias assistivas podem enfrentar dificuldades tanto no aeroporto, antes do embarque e depois do desembarque, como dentro das aeronaves durante o voo (SILVA et al., 2016).

\section{MÉTODO}

Este trabalho utiliza como método de pesquisa o Design Research Methodology (DRM) proposto por Blessing e Chakrabarti (2009). Esta pesquisa teve suas etapas de execução baseadas na proposta do DRM e foi estruturada em quatro etapas: Research Clarification, Descriptive Study I (DS I), Prescriptive Study (PS) e Descriptive Study II (DS II).

A etapa Research Clarification corresponde à revisão bibliográfica. A etapa DS I se refere à análise de dois projetos, enquanto a etapa PS corresponde à proposta do modelo com base nas informações coletadas nas etapas anteriores. A etapa DS II não é contemplada neste artigo.

\section{RESULTADOS E ANÁLISE}

\subsection{Descriptive Study I}

Nesta etapa são analisados dois projetos. O primeiro projeto foi avaliado a partir de análise documental e entrevista com os participantes, uma vez que foi realizado antes do início deste trabalho de pesquisa. No segundo projeto, o pesquisador teve participação ativa, trabalhando como gestor do projeto, financiado através de um edital do CNPq.

O primeiro projeto teve duração de oito meses e foi realizado com a participação de três alunos de graduação (uma aluna de design, dois alunos da engenharia de produção e um aluno da engenharia de computação). O projeto foi dividido em cinco etapas: Critical Experience Prototype e Critical Functional Prototype (CFP), Dark Horse, Funktional; Functional; e Final Prototype. O tema deste projeto era o "desenvolvimento de artefatos e de processos para aprimorar a experiência de voo de pessoas com deficiência e mobilidade reduzida no transporte aéreo", portanto possuía um escopo inicial do projeto era abrangente. Somente após a etapa do Dark Horse que o escopo foi definido a partir da escolha do protótipo a ser desenvolvido. Neste caso, o protótipo escolhido foi uma cadeira de rodas que facilitasse o embarque de passageiros cadeirantes tetraplégicos. Como resultado final, foi criada uma cadeira de rodas com a funcionalidade de um assento deslizante, que se desliza para o assento da aeronave, eliminando assim uma das duas transferências que o passageiro faria com uma cadeira de embarque tradicional.

O segundo projeto teve duração de doze meses e contou com uma equipe de seis alunos (dois de engenharia elétrica, um de engenharia naval, um de engenharia mecânica, e duas alunos de design). O projeto foi dividido em quatro etapas: Critical Experience Prototype e Critical Functional Prototype (CFP), Dark Horse, Funktional; e Final Prototype. O escopo deste projeto já contemplava o desenvolvimento de uma cadeira de rodas para embarque e desembarque. A cadeira do projeto anterior foi avaliada na etapa inicial e algumas de suas funcionalidades foram consideradas. Após interações dos alunos com usuários de cadeiras de rodas, comissários de bordo e funcionários de companhias aéreas, foram realizadas atividades de ideação e prototipagem nas etapas CFP e Dark Horse, de forma que que foram definidas as funcionalidades do protótipo final. 


\subsection{Prescriptive Study}

Na etapa de estudo descritivo é proposto o modelo para o desenvolvimento conceitual de produtos de TA para o setor de transporte aéreo.

Como resultado da etapa anterior, a Tabela 1 mostra algumas características relevantes para o desenvolvimento de produtos de TA que foram identificadas, tanto a partir da literatura quanto a partir dos projetos analisados em DS I. A lista de características apresentadas é levada em consideração para a criação do modelo específico desenvolvimento de produtos de tecnologia assistiva para o setor de transporte aéreo, que é mostrado na próxima sessão.

Tabela 1. Compilação das características levantadas para o desenvolvimento de produtos de TA

\begin{tabular}{|c|c|c|}
\hline CARACTERÍSTICAS REFERENTES A PRODUTOS DE T.A. & ORIGEM & PROJETOS \\
\hline Variação de condições entre pessoas com o mesmo tipo de deficiência & Literatura / Projetos & $\begin{array}{l}\text { Projeto } 1 \\
\text { Projeto } 2\end{array}$ \\
\hline $\begin{array}{l}\text { Muitas especificidades devido à diversidade de deficiências e características individuais } \\
\text { das } P C D\end{array}$ & Literatura/ Projetos & $\begin{array}{l}\text { Projeto } 1 \\
\text { Projeto } 2\end{array}$ \\
\hline Baixa escala de produção & Literatura & - \\
\hline $\begin{array}{l}\text { Indícios de uma boa aplicabilidade do design thinking no desenvolvimento de soluções de } \\
\text { TA }\end{array}$ & Literatura / Projetos & $\begin{array}{l}\text { Projeto } 1 \\
\text { Projeto } 2\end{array}$ \\
\hline Necessidade obrigatória de um bom entendimento do usuário & Literatura / Projetos & $\begin{array}{l}\text { Projeto } 1 \\
\text { Projeto } 2\end{array}$ \\
\hline Participação de usuários nas etapas iniciais de projeto & Literatura / Projetos & $\begin{array}{l}\text { Projeto } 1 \\
\text { Projeto } 2\end{array}$ \\
\hline Participação do usuário nos inúmeros testes e avaliações & Literatura / Projetos & Projeto 2 \\
\hline Empatia pelo usuário e pela solução escolhida & Projetos & $\begin{array}{l}\text { Projeto } 1 \\
\text { Projeto } 2\end{array}$ \\
\hline Equipes de projeto multidisciplinares & Projetos & $\begin{array}{l}\text { Projeto } 1 \\
\text { Projeto } 2\end{array}$ \\
\hline $\begin{array}{l}\text { Diversos ciclos de prototipagem aceleram a descoberta de falhas e contribuem para } \\
\text { melhores soluções em prazo menor }\end{array}$ & Literatura / Projetos & Projeto 1 \\
\hline $\begin{array}{l}\text { Prototipagem rápida dá agilidade e facilita a personalização / customização em tecnologias } \\
\text { assistivas }\end{array}$ & Literatura / Projetos & Projeto 2 \\
\hline Dependência de normas técnicas e regulamentações & Literatura / Projetos & $\begin{array}{l}\text { Projeto } 1 \\
\text { Projeto } 2\end{array}$ \\
\hline $\begin{array}{l}\text { No setor de transporte aéreo, dependência de normas internacionais e resoluções } \\
\text { normativas de órgãos reguladores governamentais e entidades do setor de aviação }\end{array}$ & Literatura / Projetos & $\begin{array}{l}\text { Projeto } 1 \\
\text { Projeto } 2\end{array}$ \\
\hline
\end{tabular}

Conforme proposto por Rozenfeld et al. (2006) e mostrado em algumas das obras encontradas na revisão de literatura deste trabalho, um modelo de referência pode dar origem a modelos específicos para um tipo de indústria, para uma empresa ou para um processo. $\mathrm{O}$ modelo do PDP Unificado, como modelo genérico, não possui especificidades de uma determinada indústria ou setor. Contudo, pode ser adaptado conforme questões específicas de um cenário com atividades específicas da indústria, empresa ou processo em questão.

No caso do modelo de desenvolvimento de produtos de TA, o modelo deve conter etapas e atividades referentes ao desenvolvimento específico de produtos de TA, que em outras indústrias ou setores podem ser desnecessários ou de baixa relevância. Da mesma forma, em se tratando do desenvolvimento de soluções para o setor de transporte aéreo, o modelo deve conter etapas e atividades específicas do setor.

A Figura 1 ilustra identifica as fases do modelo do PDP (2, 3 e 4 na figura) que podem corresponder às fases, etapas e atividades desenvolvidas nos projetos de TA analisados. Considerando que o desenvolvimento de produtos de TA exige uma proximidade maior com o usuário, o uso de uma abordagem centrada no usuário seria o ideal. 
Figura 1. Fases do modelo do PDP Unificado. Fonte: Adaptado de Rozenfeld et al. (2006)
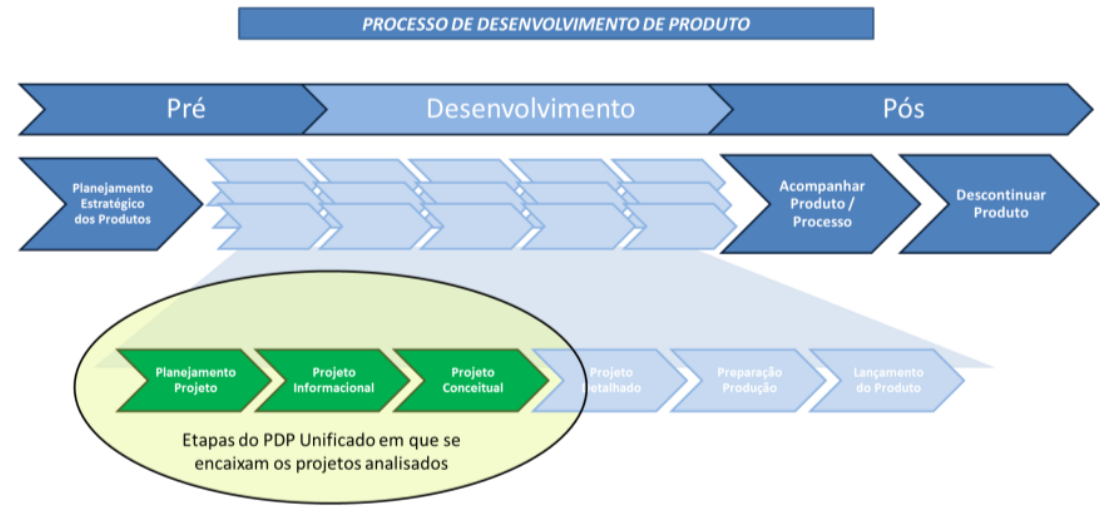

Dessa forma, a abordagem do design thinking pode ser adotada para o desenvolvimento de produtos de TA, com uso das etapas do DT como etapas de projeto. Assim, o modelo do PDP de TA passa a contemplar uma visão com foco no usuário ao longo das fases e etapas do processo.

O modelo proposto contempla etapas referentes ao desenvolvimento de conceitos de produtos no âmbito de ambientes de pesquisa. Dessa forma, corresponde ao que seriam as três etapas do PDP Unificado mostradas na Figura 1. Em sua substituição, o modelo propõe as seguintes três fases: Avaliação e entendimento do usuário; Desenvolvimento de conceitos; Prototipagem final.

Figura 2. Proposta das fases e etapas do modelo. Fonte: criado pelo autor

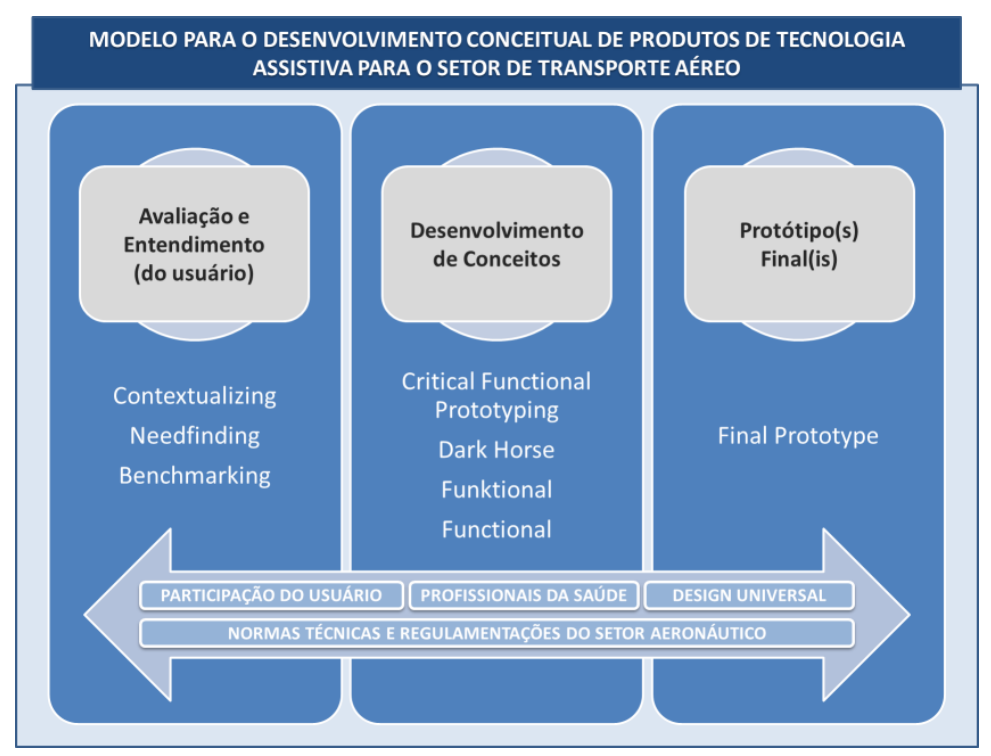

As fases contemplam etapas de DT baseadas na proposta da d.School (2009). A primeira fase envolve atividades de contextualização, entendimento do usuário e busca de soluções já publicadas e/ou existentes no mercado. As fases seguintes correspondem às atividades de design thinking até a prototipagem final. A segunda fase corresponde às primeiras etapas de geração do conceito funcional (CFP), a etapa de Dark Horse, e as etapas de desenvolvimento de funcionalidades (Funktional e Functional). As quatro etapas envolvem diversos ciclos de ideação, prototipagem e teste. A terceira fase corresponde ao desenvolvimento do protótipo final do conceito, que deve incluir as funcionalidades prototipadas, testadas e escolhidas nas etapas anteriores. A Figura 2 ilustra as fases e etapas de forma linear. 
O modelo deve incluir a participação do usuário final em todas as etapas do projeto, de forma que seus feedbacks e opiniões possam contribuir para o desenvolvimento de uma solução com maior usabilidade e utilidade. Além disso, é recomendado também o envolvimento de profissionais da área da saúde, como fisioterapeutas e terapeutas ocupacionais, que estejam em contato direto e constante com os usuários finais. Esta interação também pode contribuir para o desenvolvimento de uma solução mais adequada para o usuário final.

Além de normas técnicas sobre o desenvolvimento de produtos específicos, como, por exemplo, normas sobre cadeiras de rodas de órgãos como o INMETRO no Brasil, devem também ser consideradas as normas e regulamentações específicas do setor aeronáutico. As normas da ANAC, da INFRAERO e da IATA devem ser consideradas, uma vez que regem as atividades do setor e contém especificidades para os ambientes de aeroportos e ambiente interno das aeronaves. Por fim, o modelo contempla que o conceito de design universal ao longo do projeto.

\section{CONCLUSÃO}

Com base na literatura e nas análises dos três projetos em DS I, tem-se um embasamento para identificar quais etapas do PDP são relevantes para o desenvolvimento de produtos de TA, no contexto de projetos em ambiente de pesquisa. A literatura e os projetos avaliados forneceram informações para o entendimento de como funciona o processo de DP de TA. A partir da revisão de literatura fica claro que as soluções de tecnologia assistiva muitas vezes são únicas, e nesses casos não demandam produção em larga escala. Características importantes foram identificadas na literatura e verificadas nos projetos analisados, como a necessidade de proximidade do usuário, tanto na etapa de needfinding quanto nas etapas seguintes nas atividades de prototipagem e avaliação dos protótipos.

O modelo em questão fica restrito ao contexto de desenvolvimento conceitual de produtos em centros de pesquisa, em grupos de pesquisa e em instituições de ensino. Dessa forma, pode ser utilizado em projetos de pesquisa, como também em disciplinas de projeto e desenvolvimento de produto que envolva design thinking. Abordagens similares já são utilizadas em cursos, como, por exemplo, o curso descrito por Zancul et al. (2016) em que foram desenvolvidos protótipos de soluções médicas em projetos de disciplina semestral. O modelo pode ser adaptado para uso tanto em disciplinas semestrais como disciplinas anuais, neste caso com prazos maiores para que os estudantes possam desenvolver o projeto.

Considerando a limitação da área dos projetos avaliados neste trabalho, o modelo pode ser objeto de trabalhos futuros para validação no desenvolvimento de produtos diferentes de tecnologia assistiva e em outros setores de aplicação diferentes de instituições de pesquisa.

\section{AGRADECIMENTOS}

Os autores agradecem o apoio do CNPq e da CAPES para a realização deste trabalho.

\section{REFERÊNCIAS}

ABEAR. Aviação Brasileira - Agenda 2020. São Paulo, SP: Associação Brasileira das Empresas Aéreas, 2014. Disponível em: < http://www.abear.com.br/dados-e-fatos/agenda2020>. Acesso em: 19 jan. 2016.

BARGAR, C. et al. Embraccess: Redesigning the Flying Experience for Passengers with 
Limited Mobility. Stanford, CA: Stanford Digital Repository, 2014. Disponível em: <https://purl.stanford.edu/dz214cf9700>. Acesso em: 18 mar. 2016.

BLESSING, L.; CHAKRABARTI, A. DRM, a design research methodology. 1. ed. London: Springer-Verlag London, 2009. v. 1

BROWN, T. Design Thinking. Harvard Business Review, p. 11, 2008.

CLARK, K. B.; FUJIMOTO, T. Product development and competitiveness. Journal of the Japanese and International Economies, v. 6, n. 2, p. 101-143, jun. 1992.

COOK, A. M.; POLGAR, J. M. Cook and Hussey's assistive technologies: principles and practice, Third Edition. 3rd. ed. St Louis, Missouri: Elsevier Inc., 2008.

D.SCHOOL. Bootcamp Bootleg. Stanford: Hasso Platner Institute of Design at Stanford, 2009.

FLAGG, J. L. et al. Need to Knowledge (NtK) Model: an evidence-based framework for generating technological innovations with socio-economic impacts. Implementation Science, v. 8, n. 1, p. 21, 15 dez. 2013.

HERSH, M. A. The Design and Evaluation of Assistive Technology Products and Devices Part 1: Design. In: International Encyclopedia of Rehabilitation. Buffalo: Center for International Rehabilitation Research Information and Exchange (CIRRIE), 2010.

IATA. IATA Fact Sheet: Industry Statistics. Miami: International Air Transport Association, 2016. Disponível em: < https://goo.gl/roYXra >. Acesso em: 17 nov. 2016.

KUTZ, M. Standard Handbook of Biomedical Engineering and Design. NY: [s.n.].

MACE, R. L. Universal design in housing. Assistive technology : the official journal of RESNA, v. 10, n. 1, p. 21-8, 22 jan. 1998.

MORETTI, I. C. Modelos de referência para desenvolvimento de produtos: análise da contribuição acadêmica. Anais XXXII ENEGEP. Anais...Rio de Janeiro: 2012. Disponível em: <http://www.abepro.org.br/biblioteca/enegep2012_TN_STO_161_940_19867.pdf>. Acesso em: 2 fev. 2016

NAIARA, T. et al. Brazilian air transport: experiences of elderly passengers. Proceedings 19th Triennial Congress of the IEA. Anais...Melbourne: 2015. Disponível em: <http://www.iea.cc/congress/2015/654.pdf>. Acesso em: 17 fev. 2017

OKUMURA, M. L. M.; CANCIGLIERI JUNIOR, O. A Engenharia Simultânea aplicada no desenvolvimento de produtos inclusivos: uma proposta de framework conceitual. $\mathbf{8}^{\circ}$ Congresso Brasileiro de Gestão de Desenvolvimento de Produto - CBGDP, 2011.

RAYMAN, R. B. Passenger safety, health, and comfort: a review. Aviation, space, and environmental medicine, v. 68, n. 5, p. 432-40, maio 1997.

ROZENFELD, $\mathrm{H}$. et al. Gestão de desenvolvimento de produtos: uma referência para a melhoria do processo. São Paulo: Editora Saraiva, 2006.

WORLD BANK GROUP. Air transport - passengers carried in World. 2015. Disponível em: < http://data.worldbank.org/indicator/IS.AIR.PSGR >. Acesso em: 30 jan. 2016.

ZANCUL, E. DE S. et al. Students' engagement in the implementation of results obtained in capstone design courses: A preliminary model for detailed design and go-tomarket activities. Proceedings of the 18th International Conference on Engineering and Product Design Education, E\&PDE 2016. Anais...Aalborg: 2016 